\title{
Facilitando el abordaje de delirium y síndrome de caídas mediante Aprendizaje Basado en Problemas en estudiantes de Medicina en una universidad colombiana
}

\section{Teaching the approach to delirium and fall syndrome through Problem-Based Learning in Medicine students at a Colombian university}

\author{
Miguel Oswaldo Cadena-Sanabria ${ }^{1,2 *}$ Sonia Ortiz Ruiz ${ }^{1}$, Sergio Serrano Gómez ${ }^{1}$ \\ 1 Universidad Autónoma de Bucaramanga, Colombia; mcadena341@unab.edu.co; ORCID ID: 0000-0001- \\ 9807-3029 \\ 2 Universidad Industrial de Santander, Colombia \\ * Correspondencia: mcadena341@unab.edu.co
}

Recibido: 4/1/2021; Aceptado: 1/3/2021; Publicado: 8/3/2021

Resumen: La formación de Grado en Geriatría en Colombia es limitada. El corto tiempo de rotaciones obliga a adoptar estrategias didácticas eficientes. Se diseñó un estudio para evaluar el desarrollo de competencias mediante una estrategia de aprendizaje basado en problemas (ABP) que se comparó con la metodología tradicional. Se incluyó a estudiantes de grado que iniciaban la rotación de Geriatría y que fueron asignados a una estrategia de ABP o de seminarios. Se definieron competencias para el abordaje de delirium y caídas/osteoporosis. El desarrollo de las competencias se evaluó a través de una Evaluación Clínica Objetiva Estructurada (ECOE) y prueba escrita, a la que optaron 18 estudiantes, de los que un 55.6\% son mujeres. La prueba escrita final en el grupo ABP fue de 4.02 vs 3.02 en control ( $p=0.005)$. La puntuación ECOE en el grupo ABP fue de 2.72 vs 2.03 en control $(p=0,062)$. Solo 4 estudiantes (pertenecientes al grupo de ABP) aprobaron la ECOE. La competencia de trabajo interprofesional tuvo mayor desarrollo en el grupo ABP (3.58 vs 2.04, p=0.005). La apreciación respecto a la realización de seminarios es que solo demanda conocimiento memorístico, mientras que el ABP fortalece el desarrollo de competencias cognitivas y el trabajo colaborativo. Respecto a la técnica de evaluación ECOE, se resalta que enfrenta al estudiante con un ambiente más cercano al ejercicio profesional. El ABP favoreció un mayor desarrollo de competencias en Geriatría tanto en la prueba escrita como en la ECOE. Hubo una mejor resolución de las estaciones concernientes a capacidad de interrogatorio, realización de pruebas funcionales y trabajo interprofesional.

Palabras clave: ABP, geriatría, educación médica, competencias, delirium, caídas, ECOE

\begin{abstract}
Undergraduate Geriatrics training in Colombia is still limited. The short rotation time forces to adopt efficient teaching strategies. A study was designed to assess the development of skills through a problem-based learning strategy compared to the traditional methodology. Cross sectional study. Undergraduate students who started Geriatrics rotation were randomized to an PBL strategy (intervention) or seminars (control). Skills were defined to address delirium and falls / osteoporosis. The development of skills was evaluated through OSCE and written test in 18 students, $55.6 \%$ women. The final written test in the PBL group was 4.02 vs. 3.02 in control $(\mathrm{p}=0.005)$. The OSCE score in the PBL group was 2.72 vs. 2.03 in control $(\mathrm{p}=0.062)$. Only 4 students (belonging to the PBL group) approved the OSCE. The interprofessional work skill had greater development in the PBL group (3.58 vs. 2.04, $\mathrm{p}=0.005$ ). The appreciation regarding the realization of seminars is that it only demands memorial knowledge whereas PBL strengthens the development of cognitive skills and collaborative work. Regarding the OSCE evaluation technique, it was highlighted that the student faces an environment closer to the professional practice. The PBL favored a greater development of skills in Geriatrics both in the written test and
\end{abstract}


in the OSCE. There was a better resolution of the stations concerning interrogation capacity, performance of functional tests and interprofessional work.

Keywords: Problem-based learning, geriatrics, medical education, skills, delirium, falls, OSCE

\section{Introducción}

El envejecimiento poblacional a nivel mundial ha transformado la dinámica de atención médica y por ende ha exigido un cambio en los objetivos de formación del talento humano en salud. Desafortunadamente, los médicos de atención primaria carecen aún de competencias integrales para la atención de problemas de pacientes mayores como el delirium y el síndrome de caídas (1). La inclusión de cátedras de Geriatría y el recurso docente en la mayoría de universidades en el mundo aún es escaso. Solamente un $25 \%$ de las universidades en España para 2015 formulaban competencias en relación a geriatría dentro de su currículo (2). En Colombia el panorama estaba alrededor del $20 \%$ de las facultades de Medicina (3). El tiempo de rotaciones en especialidades clínicas y quirúrgicas es reducido a semanas, lo cual obliga a la adopción de estrategias didácticas más eficientes que las tradicionales clases magistrales, revisión de casos o historias clínicas, rondas docente asistenciales y seminarios de diapositivas empleados en estas asignaturas.

El aprendizaje basado en problemas (ABP) ha demostrado de manera consistente un mayor impacto en la adquisición de habilidades y conocimientos para el ejercicio profesional del médico comparado con otras estrategias didácticas (4). Existen experiencias del campo de acción del ABP en la docencia de la geriatría a nivel mundial (5). Las técnicas de evaluación en medicina clínica son variadas y modifican notoriamente la evidencia que pueda soportar diferencias entre el ABP y otras estrategias. En Colombia se ha empleado ABP como enfoque curricular en facultades de Medicina (6) y como estrategia didáctica en asignaturas de pregrado de fisiología (7) y farmacología (8). En este último estudio, la evaluación comparativa se limitó a pruebas cognitivas y simulación de casos. El examen o evaluación clínico objetivo estructurado (ECOE) ha representado una herramienta válida para verificar las competencias clínicas y el desempeño en estudiantes y profesionales en Medicina. (9) (10).

A lfecha de hoy, no existen en Colombia publicaciones que evidencien el papel del ABP en la enseñanza de la Geriatría y asimismo de la ECOE como técnica para evaluar el desarrollo de las competencias en relación al adulto mayor. Por tal razón, se planteó el presente estudio cuyo objetivo fue evaluar el impacto de una estrategia de ABP comparada con la estrategia de seminarios en el aprendizaje significativo de dos grandes síndromes geriátricos (delirium y caídas/osteosarcopenia) en estudiantes de pregrado de Medicina de la Universidad Autónoma de Bucaramanga (UNAB).

\section{Métodos}

Se diseñó un estudio descriptivo donde se incluyeron estudiantes de sexto semestre de Medicina que iniciaban la rotación de Geriatría clínica y recibieron una estrategia de ABP o una estrategia de seminarios tipo ponencia. Se diseñaron las competencias en medicina geriátrica para el abordaje de dos problemas clínicos (delirium y caídas/osteoporosis), desde las recomendaciones latinoamericanas (11) y siguiendo el enfoque socioformativo (12).

Se dividieron los estudiantes en 2 grupos de 4-5 personas para el grupo de intervención y 2 grupos de similar cantidad para el grupo control. Los 4 grupos recibieron igualmente dos clases magistrales, cursaron la rotación en el servicio clínico de Medicina Interna y Geriatría en una institución de III nivel de la ciudad de Bucaramanga, Colombia. En ella, 
realizaron la historia clínica de pacientes hospitalizados, participaron de la revisión docente asistencial y asistieron a consulta externa. Durante estas actividades tuvieron la posibilidad de aplicar pruebas de tamizaje cognitivo, nutricional y evaluación funcional. La duración de la rotación de geriatría clínica en cada grupo tuvo una duración de 2 semanas.

\section{Metodología ABP:}

Los estudiantes de este grupo desarrollaron 2 secuencias didácticas de ABP, una para el abordaje del delirium y otra para el abordaje de caídas y osteoporosis. La primera semana se trabajó el problema de delirium y la segunda el problema de caídas y osteoporosis/sarcopenia. Cada una de las sesiones tuvo dos momentos de encuentro, uno inicial y otro final de sustentación y desarrollo del problema. En el intermedio de las sesiones grupales, los estudiantes tuvieron un encuentro de 1 hora con una tutora para aclarar algunas inquietudes sobres los aspectos que habían investigado y además recibir orientaciones sobre las pruebas o resultados que venían integrados al problema planteado.

\section{Metodología seminarios:}

El grupo desarrolló los contenidos y competencias a través de la presentación de seminario tipo ponencia. Emplearon diapositivas en Power Point. La presentación de cada seminario tuvo una duración de 1 hora. Se realizaron intervenciones de manera intermitente por parte del docente para generar espacios de reflexión y realimentación en torno a los temas abordados de delirium y caídas.

El desarrollo de las competencias se evaluó a través de un examen clínico objetivo estructurado (ECOE) (ver tabla 1) y una prueba escrita pre y post-test. Al finalizar la última reunión de la segunda semana de rotación, se procedió a aplicar la prueba escrita final. La puntuación se registró en una nota cuantitativa de 0 a 5. Posteriormente, los estudiantes fueron citados para la realización del examen ECOE dos semanas después de finalizada la rotación.

Tabla 1. Examen Clínico Objetivo Estructurado (ECOE) _ Geriatría UNAB

\begin{tabular}{|c|l|l|l|}
\hline Estación & Competencia & \multicolumn{1}{|c|}{ Criterio } & \multicolumn{1}{|c|}{ Actividad } \\
\hline 1 & Interrogatorio & $\begin{array}{l}\text { Identifica de forma clara motivo de } \\
\text { consulta, enfermedad actual e infor- } \\
\text { mación de antecedentes médicos del } \\
\text { paciente }\end{array}$ & $\begin{array}{l}\text { Entrevista a hija (actriz) de } \\
\text { paciente (maniquí) en consul- } \\
\text { torio }\end{array}$ \\
\hline 2 & Juicio clínico & $\begin{array}{l}\text { Interpreta de manera adecuada set } \\
\text { de paraclínicos de un paciente con } \\
\text { delirium y argumenta plan diagnós- } \\
\text { tico y terapéutico }\end{array}$ & $\begin{array}{l}\text { Identificación de alteraciones } \\
\text { paraclínicas, plan de trata- } \\
\text { miento que incluye SS3\%, for- } \\
\text { mulación de haloperidol }\end{array}$ \\
\hline 3 & $\begin{array}{l}\text { Evaluación } \\
\text { funcional } \\
\text { sempeño físi- } \\
\text { co) }\end{array}$ & $\begin{array}{l}\text { Evalúa el desempeño físico de una } \\
\text { persona a través de la realización de } \\
\text { la prueba SPPB y velocidad de la } \\
\text { marcha. Interpreta los resultados y } \\
\text { prescribe un plan de ejercicio físico. }\end{array}$ & $\begin{array}{l}\text { Cálculo e interpretación de la } \\
\text { velocidad de la marcha y } \\
\text { SPPB. Prescripción de ejerci- } \\
\text { cio }\end{array}$ \\
\hline 4 & Terapéutica & $\begin{array}{l}\text { Interpreta una densitometría ósea y } \\
\text { planifica un tratamiento efectivo y } \\
\text { seguro para osteoporosis }\end{array}$ & $\begin{array}{l}\text { Lectura de densitometría de } \\
\text { una paciente en consultorio. } \\
\text { Fórmula médica con prescrip- } \\
\text { ción de antiresortivo o terapia }\end{array}$ \\
\hline
\end{tabular}




\begin{tabular}{|c|c|c|c|}
\hline & & & anabólica para osteoporosis \\
\hline 5 & $\begin{array}{l}\text { Trabajo inter- } \\
\text { profesional }\end{array}$ & $\begin{array}{l}\text { Valora el papel de terapia física, } \\
\text { ocupacional y enfermería en el ma- } \\
\text { nejo integral de un paciente anciano } \\
\text { hospitalizado. Construye un plan de } \\
\text { tratamiento que incluye las medidas } \\
\text { no farmacológicas para delirium y } \\
\text { rehabilitación }\end{array}$ & $\begin{array}{l}\text { Ronda simulada de servicio } \\
\text { de orto geriatría: discusión in- } \\
\text { terdisciplinaria, formulación } \\
\text { de medidas de rehabilitación } \\
\text { y estimulación cognitiva }\end{array}$ \\
\hline
\end{tabular}

Validación de instrumentos y análisis estadístico

Se realizó un proceso de validación (13) de los instrumentos de evaluación, tomando como expertos 2 docentes de Geriatría de las principales facultades de Medicina del país (Universidad Nacional, Pontificia Universidad Javeriana) y 1 docente de Geriatría de Perú. Se definió la validez de contenido, el grado de comprensibilidad de cada una de las preguntas y su pertinencia. Se incluyeron variables sociodemográficas, desempeño académico previo y autoevaluación. Se aplicaron técnicas de estadística descriptiva y análisis para variables cuantitativas según su distribución. Se aplicaron pruebas de significancia estadística t test o test de Fischer según el tipo de variable. La base de datos se analizó usando el Software Stata 12.0. Se consideró un valor de p de 0.05 o menos para diferencias significativas. Asimismo, el investigador principal realizó una entrevista semiestructurada, al finalizar la ECOE, a los estudiantes y un grupo focal para evaluar percepciones de los evaluadores sobre la estrategia didáctica y técnica de evaluación.

\section{Consideraciones éticas}

La presente investigación respetó los aspectos éticos enmarcados en la resolución 8430 de 1993 de Colombia. Se solicitó consentimiento informado escrito para la inclusión en el estudio. El tratamiento de los datos personales evitó la identificación de cada uno de los participantes. Se respetó la dignidad y los principios bioéticos de beneficencia, autonomía y no maleficencia. Asimismo, contó con el aval formal de la decanatura del programa de Medicina UNAB y el comité de ética institucional.

\section{Resultados}

Un total de 18 estudiantes se incluyeron en el estudio, de los cuales se realizó una asignación por grupo de rotación a la estrategia de ABP y al grupo control (seminario). Cada estrategia didáctica tuvo un número de 9 estudiantes. Del grupo seminario se señalan dos pérdidas debido a que estos estudiantes no asistieron al examen ECOE. La edad media fue de 21 años (19-24) y un 55.6\% fueron mujeres. Las características generales de la población se describen en la tabla 2. El promedio académico previo de los estudiantes no tuvo diferencias estadísticamente significativas entre los dos grupos. Los estudiantes que se encontraban en el grupo control tenían un promedio de carrera de 3.6 y los del grupo ABP de 3.7 (p=0.61). Situación similar ocurrió con el resultado del núcleo integrador (examen promocional realizado en quinto semestre sobre los contenidos trabajados durante los semestres iniciales de carrera) en cada uno de los grupos (3.6 vs 3.5). Se logró una asistencia del $100 \%$ a todas las sesiones y reuniones del ABP y de seminarios.

Tabla 2. Características generales de los participantes en cada uno de los grupos de estudio.

\begin{tabular}{|c|c|c|}
\hline Variable & Grupo ABP (n=9) & Grupo seminario (n=9) \\
\hline Edad (años) & 21.3 & 20.6 \\
\hline Género femenino & $55.6 \%(5)$ & $55.6 \%(5)$ \\
\hline
\end{tabular}




\begin{tabular}{|c|c|c|}
\hline \multicolumn{3}{|c|}{ Estado civil } \\
\hline Soltero & $88.8 \%(8)$ & $100 \%(9)$ \\
\hline Unión libre & $11.2 \%(1)$ & 0 \\
\hline \multicolumn{3}{|c|}{ Estrato socioeconómico (3-4, clase media; 5-6, clase alta) } \\
\hline 3 & 0 & $22.2 \%(2)$ \\
\hline 4 & $55.6 \%(5)$ & $11.1 \%(1)$ \\
\hline 5 & $22.2 \%(2)$ & $44.5 \%(4)$ \\
\hline 6 & $22.2 \%(2)$ & $22.2 \%(2)$ \\
\hline Promedio carrera $(/ 5)$ & 3.7 & 3.65 \\
\hline Núcleo integrador* $(/ 5)$ & 3.6 & 3.5 \\
\hline \multicolumn{3}{|c|}{ Semestres perdidos } \\
\hline Ninguno & $44.4 \%(4)$ & $44.4 \%(4)$ \\
\hline Uno & 0 & $11.1 \%(1)$ \\
\hline Dos o más & $55.6 \%(5)$ & $44.4 \%(4)$ \\
\hline \multicolumn{3}{|c|}{ Horas repaso semanal } \\
\hline Menos de 2 horas & $11.1 \%(1)$ & 0 \\
\hline Entre 2-4 horas & $33.3 \%(3)$ & $33.3 \%(3)$ \\
\hline 4-8 horas & $11.1 \%(1)$ & $33.3 \%(3)$ \\
\hline Más de 8 horas & $44.5 \%(4)$ & $33.3 \%(3)$ \\
\hline \multicolumn{3}{|c|}{ Estrategias de aprendizaje } \\
\hline No conoce & $44.4 \%(4)$ & $44.4 \%(4)$ \\
\hline Aplica alguna & $44.4 \%(4)$ & $55.6 \%(5)$ \\
\hline \multicolumn{3}{|c|}{ Organizadores gráficos ** } \\
\hline No emplea & $22.2 \%(2)$ & $22.2 \%(2)$ \\
\hline Emplea uno o más & $77.8 \%(7)$ & $77.8 \%(7)$ \\
\hline
\end{tabular}

*, examen que los estudiantes de $\mathrm{V}$ semestre pasan, promocional, que reúne los conceptos y conocimientos que se espera que hayan acumulado en las areas básicas de la carrera de Medicina; **, herramientas pedagógicas que permiten al estudiante consolidar y resumir ciertos conceptos de manera ilustrativa (p.ej., mapa mental, mapa conceptual, cuadro sinóptico...).

Evaluación escrita: el resultado de la prueba escrita final en el grupo control fue de 3.02 (IC del $95 \%, 2.6-3.4)$ vs 4.02 (IC del 95\%, 3.4 - 4.6) en el grupo de ABP ( $\mathrm{p}=0.005)$.

ECOE: el resultado promedio de la ECOE fue de 2.41, con una desviación estándar de 0.74 . La mediana estuvo en 2.32. La mayor puntuación fue 3.82 y la menor, 1.08. El desempeño en el grupo de ABP fue de 2.72 (mínimo de 1.34, máximo de 3.82) y en el grupo control fue de 2.03 (mínimo de 1.08, máximo de 2.64) (p: 0.062). Solo 4 estudiantes lograron aprobar la ECOE, todos del grupo ABP. La competencia de trabajo interprofesional tuvo mayor desarrollo en el grupo ABP (3.58 vs 2.04, $\mathrm{p}=0.005)$. Dentro de las otras competencias profesionales evaluadas, no se encontraron diferencias significativas (ver figura 1). Es importante mencionar que el nivel de dominio de las competencias reflejado en la ECOE estuvo predominantemente en básico y autónomo, contrastando un poco con la calificación obtenida en la prueba escrita, la cual estuvo en promedio en 4.02 en el grupo de intervención. Esta diferencia en el resultado reafirma la importancia de modificar la forma de evaluación de competencias clínicas en profesionales de la salud. 
La entrevista semiestructurada se realizó al finalizar el examen ECOE. La entrevista inició con la presentación de la actividad por parte del docente y tuvo una duración de 5 minutos por estudiante. El docente realizó las anotaciones correspondientes con relación a las respuestas de los estudiantes. Las preguntas realizadas fueron:

1. ¿Cómo se organizó el grupo para la preparación de las sesiones de aprendizaje basado en problemas/seminario? Distribución de tareas, preparación teórica, organización de las respuestas al problema

2. ¿Qué percepción tiene sobre el aprendizaje logrado con la actividad (ABP/seminario) del tema desarrollado (delirium y caídas)?

3. ¿Cómo cree que preparar y presentar un tema en forma de $\mathrm{ABP} /$ seminario permite el aprendizaje del tema abordado? Señale fortalezas o debilidades que percibe de esta metodología

4. ¿Qué exigencias o retos demandó para usted la preparación de las sesiones de $\mathrm{ABP} /$ seminario? Se entrevistaron en total 6 estudiantes.

La apreciación en general respecto a la realización de seminarios es que solo demanda conocimiento memorístico. Los estudiantes mencionaron "es coger lo del libro", "es recitar lo que está". El ABP fortalece el desarrollo de competencias cognitivas y trabajo colaborativo: "la fortaleza es que se revisa uno o varios temas dependiendo del caso, se aplica lo que se haya leído al caso y al ser en grupo todos pueden aportar ideas diferentes", "el mayor reto fue trabajar en equipo, no todas las personas están dispuestas a reunirse para desarrollar un caso, prefieren utilizar herramientas electrónicas para esto, como desarrollarlo por Google drive. Esto hace que sea difícil llegar a una conclusión grupal".

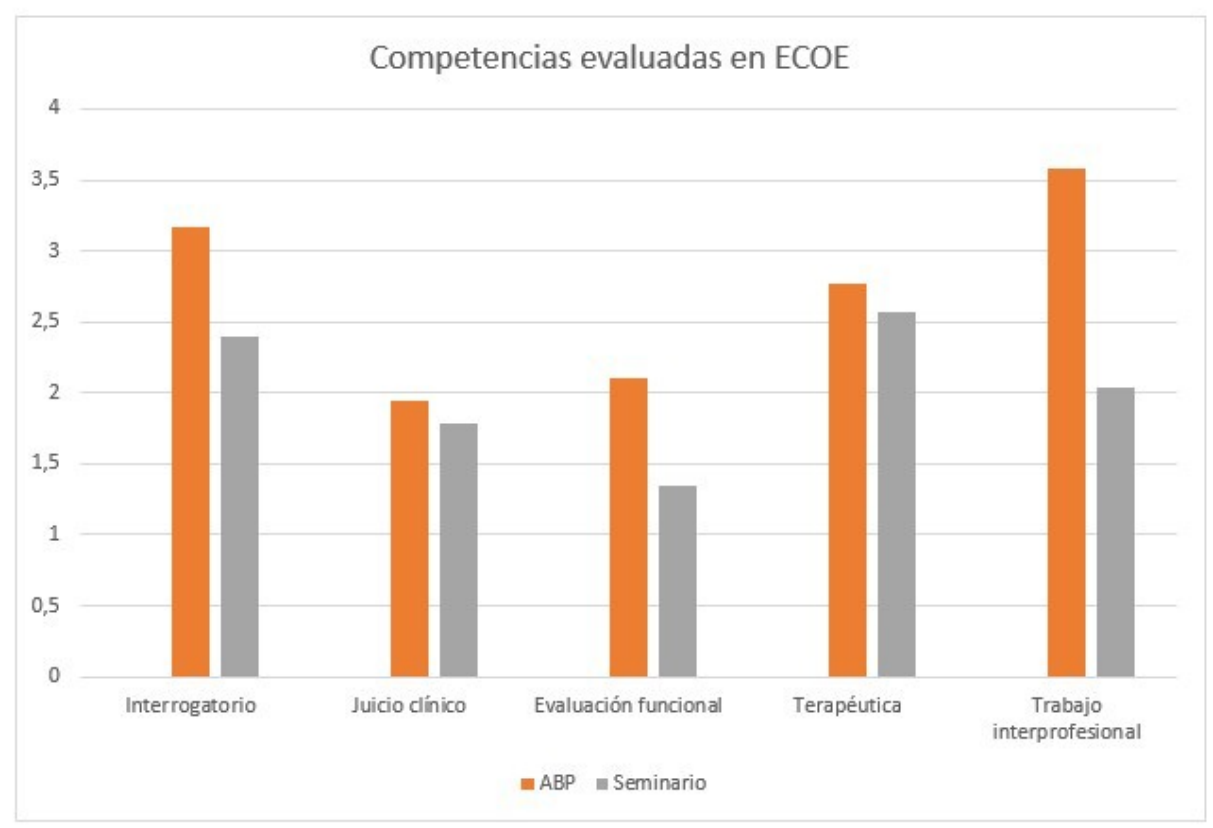

Figura 1. Resultados de la ECOE entre ABP y grupo control (interrogatorio, $p=0.09$; evaluación funcional, $\mathrm{p}=0.34$; trabajo interprofesional, $\mathrm{p}=0.005$ ).

Respecto a la técnica de evaluación ECOE se resaltó que lo enfrenta a un ambiente más cercano al que enfrentará en su ejercicio profesional. Los evaluadores del ECOE en el grupo focal resaltaron: "El ECOE es un examen objetivo, se define claramente lo que el 
estudiante debe saber y evalúa de manera estandarizada ese conocimiento o habilidad", "evalúa el hacer, siempre evaluamos el saber únicamente", "una fortaleza muy importante es que cada estación brindaba un espacio de realimentación al estudiante, para que identificara sus debilidades o falencias".

\section{Discusión}

Se evidenció un mayor resultado promedio en la prueba final de conocimientos en los estudiantes que pertenecieron al grupo de $\mathrm{ABP}$, comparado con el grupo control. Es decir, un mejor nivel de desempeño a nivel de pruebas del saber y saber contextualizado. La ECOE evidenció una diferencia estadísticamente significativa en el grado de desarrollo de las competencias de trabajo interprofesional e interrogatorio entre los dos grupos. Dichos resultados pueden ser explicados por las ventajas que ofrece la estrategia didáctica del $\mathrm{ABP}$ en relación al trabajo colaborativo, la autorregulación y la interacción social, exigiendo una puesta en escena de éstas por parte de los estudiantes para la resolución de problemas (14).

Una fortaleza del presente estudio es que los grupos tuvieron unas características similares al inicio de la investigación. Variables sociodemográficas como edad, promedio académico de la carrera, estado civil y semestres cursados tuvieron una distribución homogénea en ambos grupos. Esta apreciación minimiza sesgos de selección o de información que pudieran afectar en cierta medida las relaciones entre los resultados y las estrategias de didácticas.

Dentro de las limitaciones del estudio se pueden señalar la metodología descriptiva, el poco tamaño de muestra lo cual pudo haber disminuido el poder del estudio para encontrar diferencias en las demás competencias relacionadas con juicio clínico y terapéutica. Por otra parte, no se evaluaron estrategias de aprendizaje empleadas por los estudiantes de una manera sistemática. Las estrategias afectivas, de apoyo y control (motivación, metacognición) o cognitivas como las técnicas de búsqueda de información, no se indagaron en la investigación. Éstas constituyen factores personales que están asociados a la capacidad para regular procesos de aprendizaje, es decir, que pueden potenciar el aprendizaje significativo (15).

La metodología evaluada en la presente investigación y los resultados obtenidos apoyan las recientes recomendaciones para mejorar la enseñanza de la Geriatría en el grado desde la Sociedad Española de Gerontología y Geriatría (16). En 3 de ellas tienen cabida las evidencias derivadas del presente trabajo: la recomendación 8 (es recomendable incorporar nuevos métodos docentes a los programas formativos en Geriatría. Solo 4 facultades en España empleaban el modelo educativo de aprendizaje basado en problemas en el 2015); la recomendación 9 (deben incluirse progresivamente el uso de tecnologías y el examen clínico objetivo y estructurado) y la recomendación 10 (es necesario aumentar la investigación y la innovación en la docencia de Geriatría).

Abizanda Soler y cols (17) describen la experiencia de la implementación de un programa docente en Geriatría en la facultad de Medicina de la Universidad de Castilla-La Mancha en Albacete (España) durante el año 2002. Se resalta la enseñanza empleando educación basada en problemas a los alumnos de quinto año. Los grupos de estudiantes son de 10-15 y durante cursos de 6 semanas se abordan 10 problemas junto a dos seminarios complementarios. Asimismo, se acompaña dicha metodología de una rotación de 3 semanas en unidad de agudos, unidad de recuperación funcional y consulta externa. La enseñanza de los 10 problemas abarca aspectos relevantes como cuidado paliativo, trabajo interdisciplinario, niveles asistenciales, cambios fisiológicos, aspectos 
farmacológicos, ejercicio y calidad de vida. Los problemas se resuelven en dos sesiones de una hora. De manera interesante en este mismo artículo, se presenta la evaluación de la metodología por parte de los estudiantes, donde se resalta el cambio en los conocimientos de los estudiantes para optimizar el proceso asistencial en la atención del paciente anciano. Hay una mayor identificación de los pacientes que se benefician de recibir atención por la especialidad en servicios de agudos y en unidades de recuperación funcional (por ejemplo pacientes con estados confusionales agudos, fractura de cadera).

No hay referencias de experiencias del ABP en la enseñanza específica de la Geriatría en Colombia. Es necesario el diseño posterior de estudios experimentales con adecuado cálculo de tamaño de muestra para robustecer la evidencia científica en relación a las ventajas del $\mathrm{ABP}$ respecto a la didáctica basada en seminarios en educación médica concerniente a la especialidad de Geriatría (18).

\section{Conclusiones}

- Este es el primer estudio en Latinoamérica que describe un núcleo de competencias en Geriatría diseñado desde un enfoque socioformativo.

- La estrategia didáctica de ABP favoreció un mayor desarrollo de competencias en Geriatría tanto en la prueba escrita como en la evaluación clínica objetiva estructurada, comparada con el grupo de seminarios.

- Se observó mayor puntuación en el nivel de conocimientos y una mejor resolución de las estaciones concernientes a capacidad de interrogatorio, realización de pruebas funcionales y trabajo interprofesional.

- Tanto la estrategia de enseñanza como las técnicas de evaluación como la ECOE, permiten un mayor logro de competencias disciplinares y genéricas, acercando progresivamente al estudiante a su campo profesional.

Financiación: No ha habido financiación.

Declaración de conflicto of interés: Los autores declaran no tener ningún conflicto de intereses.

\section{Referencias}

1. Comisión para la Transformación de la Educación Médica en Colombia. Recomendaciones para la transformación de la educación médica en Colombia. 2017. En https://www.minsalud.gov.co/sites/rid/ Lists/BibliotecaDigital/RIDE/VS/MET/recomendaciones-comision-para-la-transformacion.pdf

2. Vilches-Moraga A, Ariño-Blasco S, Verdejo-Bravo C, Mateos-Nozal J. Plan de estudios universitarios en medicina geriátrica desarrollado utilizando una técnica internacional Delphi modificada. Rev Esp Geriatr Gerontol. 2015;50(2):82-8. http:/ / doi.org/10.1016/j.regg.2014.10.003

3. López JH, Reyes-Ortiz CA. Geriatric Education in Undergraduate and Graduate Levels in Latin America. Gerontol Geriatr Educ. 2015;36(1):3-13. http:/ / doi.org/10.1016/j.regg.2014.11.008

4. Guillamet Lloveras A. Influencia del Aprendizaje Basado en Problemas en la práctica profesional [Internet]. Universidad de Granada; 2011 [cited 2019 Jan 12]. Available from: https://hera.ugr.es/tesisugr/20514505.pdf

5. Yanamadala M, Kaprielian VS, O'Connor Grochowski C, Reed T, Heflin MT. A problem-based learning curriculum in geriatrics for medical students. Gerontol Geriatr Educ. 2016;39(2):122-31. https:/ / doi.org/10.1080/02701960.2016.1152268

6. Rodríguez Gómez HM, Lugo Agudelo LH, Aguirre Muñoz C. El aprendizaje basado en problemas, en el currículo de la Facultad de Medicina de la Universidad de Antioquia. Iatreia. 2004;17(3):245-57. Disponible en: https://revistas.udea.edu.co/index.php/iatreia/article/view/4069

7. Gonzalez Olaya HL, Galindo Cárdenas LA. Aplicación de la experiencia de aprendizaje mediado a la estrategia de aprendizaje basado en problemas, en estudiantes del tercer semestre de medicina, Universidad Autónoma de Bucaramanga, Colombia. Iatreia. 2011;24(4):422-31. Disponible en: https://revistas.udea.edu.co/index.php/iatreia/article/view/10642 
8. Niño Avendaño CA, Ospina Díaz JM, Niño Ruiz YA. Comparación entre enseñanza magistral de farmacología versus aprendizaje basado en problemas en estudiantes de Medicina, Tunja-Boyacá, 2016. Salud, Hist y Sanid On-Line. 2017; 12(3): 29-39. http://agenf.org/ojs/index.php/shs/article/download/ 255/24

9. Sureda-Demeulemeester E, Ramis-Palmer C, Sesé-Abad A. La evaluación de competencias en Medicina. Rev Clínica Española [Internet]. 2017;1-9. Available from: http://dx.doi.org/10.1016/j.rce.2017.05.004

10. Pinilla-Roa AE. Evaluación de competencias profesionales en salud. Rev Fac Med. 2013;61(1):53-70. https://revistas.unal.edu.co/index.php/revfacmed/article/view/39632

11. Cano C, Gutiérrez LM, Marín PP, Morales Martínez F, Peláez M, Rodríguez Mañas L, et al. Propuesta de contenidos mínimos para los programas docentes de pregrado en Medicina Geriátrica en América Latina. Rev Panam Salud Pública [Internet]. 2005;17(5-6):429-37. Available from: http:/ / www.scielosp.org/scielo.php?script=sci_arttext\&pid=S1020$\underline{49892005000500015 \& \operatorname{lng}=e s \& n r m=i s o \& t \operatorname{lng}=e s}$

12. García Fraile JA, López Rodríguez NM, Zuñiga R del A. Aprendizaje y Vida. Construcción, didáctica, evaluación y certificación de competencias en educación desde el enfoque socioformativo. 1st ed. Educación P, editor. México; 2014. http://revistas.ustabuca.edu.co/index.php/ESPIRAL/article/view/ $\underline{843}$

13. Castañeda Valencia CR. Diseño, validacion facial y de contenido de un instrumento de valoración de los sintomas en la mujer con enfermedad coronaria, enmarcada en la teoría de los síntomas desagradables [Internet]. 2013. Available from: http://www.bdigital.unal.edu.co/43079/

14. Abizanda Soler P, Sáez Méndez L, Cano C. Formación y docencia en Geriatría. In: España E, editor. Tratado de Medicina Geriátrica. 1st ed. Barcelona; 2015. p. 80-93. https://www.edicionesjournal.com/Papel/9788490221204/Tratado+De+Medicina+Geriatrica

15. Beltrán Llera JA. Estrategias de aprendizaje. Rev Educ. 2003;332:55-73. $\underline{\text { https: / dialnet.unirioja.es/servlet/articulo?codigo=776715 }}$

16. Mateos-Nozal J, Farré-Mercadé MV, Cruz-Jentoft AJ, Ribera Casado JM, Antón Rodrigo I, Clerencia Sierra $\mathrm{M}$, et al. Ten recommendations to improve undergraduate training in Geriatric Medicine. Rev Esp Geriatr Gerontol. 2019;54(4):203-6. http:/ / doi.org/10.1016/j.regg.2018.12.005

17. Abizanda Soler, P., Sáez Méndez, L., \& Cano, C. (2015). Formación y docencia en Geriatría. In E. España (Ed.), Tratado de Medicina Geriátrica (1st ed., pp. 80-93). Barcelona.

18. Yanamadala M, Kaprielian V, O'Connor Grochowski C, Reed T, Heflin M. A problem-based learning curriculum in geriatrics for medical students. Gerontology \& Geriatrics Education. 2016;39(2):122-131. http://doi.org/10.1080/02701960.2016.1152268

(C) 2021 por los autores. Enviado para su publicación en acceso abierto bajo los términos y condiciones de la licencia Creative Commons Attribution (CC BY) (http://creativecommons.org/licenses/by/4.0/). 